remains disputable whether and how elaborative encoding processes would generate novel associations during sleep.

\section{Dream and emotion regulation: Insight from the ancient art of memory}

\section{doi:10.1017/S0140525X13001271}

\section{Martin Desseilles ${ }^{a, b, c}$ and Catherine Duclos ${ }^{a}$ \\ ${ }^{a}$ Department of Psychology, University of Namur Medical School, B-5000 Namur, Belgium; ${ }^{\mathrm{b} C y c l o t r o n}$ Research Centre, University of Liège, B-4000 Liège, Belgium; 'Alexian Brothers Psychiatric Clinic, B-4841 Henri-Chapelle, Belgium. \\ martin.desseilles@unamur.be catherine.duclos@unamur.be}

\begin{abstract}
During dreaming, as well as during wakefulness, elaborative encoding, indexing and ancient art of memory (AAOM) techniques, such as the method of loci, may coincide with emotion regulation. These techniques shed light on the link between dreaming and emotional catharsis, post-traumatic stress disorder, supermemorization during sleep as opposed to wakefulness, and the developmental role of rapid eye movement (REM) sleep in children.
\end{abstract}

Llewellyn's thorough description of rapid eye movement (REM) dreaming as elaborative encoding for episodic memories provides a convincing argument for the value of the ancient art of memory $(\mathrm{AAOM})$ principles. Our commentary presents a complementary aspect of the dream, arguing that it is not solely "the stuff of memory," but also that of emotion regulation. Although her arguments lack an explicit link to the role of emotion in dreaming, Llewellyn does indirectly provide methods for understanding the process and purpose of emotion regulation in REM dreaming, which we explore in this commentary.

The psychological models of dreaming suggest that dreams are constructed from one's emotional history and serve partly to regulate emotions, because the dreamer is forced to look, feel, and reprocess emotional memory. During a dream, the activation of the medial prefrontal cortex would play a role in the attribution of thoughts and emotions to oneself and to characters and situations of the dream, while the deactivation of the inferior parietal regions would enable the dreamer to experience the dream in the first- and third-person perspectives. This offline role-playing would facilitate the resolution of internal conflict, which some assimilate to an emotional catharsis (Desseilles et al. 2010; 2011a; 2011b; 2012).

During lucid dreams, as in the method of loci, the dreamer constructs the dream scene and participates in the action while simultaneously being an observer. The role of the dream scene could, in certain cases, be a task of memory, memorization, recollection, and association. As an Aristotelian catharsis (Desseilles et al. 2011a), this theater-like staging could enable the dreamer to gain an outside perspective on certain behaviors, preoccupations, and problems present during wakefulness. Once lucid dreamers sufficiently identify, they could engage in mechanisms of regulation (functional or dysfunctional) of emotions, such a reappraisal, suppression, or rumination, without having to await wakefulness, as do nonlucid dreamers when they engage in the conscious regulation of emotions based on memories of dreams. The capacity of lucid dreamers to modify the course of their dreams could impact Llewellyn's theory, since she hypothesizes that following the elaborative encoding of recent memories with remote ones during REM dreams, the hippocampus may instantiate internal cortical junctions during non-REM sleep. Lucid dreamers could perhaps control elaborative encoding and thus change the quality of indexing, modifying the associations that could be recovered during wakefulness. The method of loci would therefore facilitate the regulation of emotions through an Aristotelian catharsis during the lucid dream (Zadra \& Pihl 1997) by acting directly on the mnemonic composition and hippocampal indexes. This online aspect of mnemonic composition would suggest that the use of the method of loci during wakefulness (enabling a first- or third-person perspective), whether or not combined with the induction of a lucidity during dreams (Stumbrys et al. 2012), could enable the dreamer subsequently to modulate more efficiently the emotional content associated with memories. The nonlucid dream would also play a cathartic role in emotion regulation, with REM sleep reducing the brain's reactivity to waking emotional experiences, simultaneously decreasing the intensity of previous affective experiences and diminishing subjective emotionality on the subsequent day (van der Helm et al. 2011b).

Emotion regulation could also emerge from indexing, which enables a more practical and rapid return of detailed material and facilitates the processing of new material through its anchoring to remote memories. Unsuccessful indexing would therefore lead to a more difficult return to remote memories, which is what Llewellyn suggests, and to poor processing of the material, which would linger freely. The work of bonding and indexing is particularly important in traumatic memory and post-traumatic stress disorder (PTSD), where the hypermemory of a traumatic event is present as much in dreams as in wakefulness. The recurrence of dreams linked to the traumatic event suggests that elaborative encoding and indexing are deficient. In fact, emotional hypersensitivity and endangerment of the self could inhibit elaborative encoding through hyperassociations to other memory episodes, leading to an absence of bonding. The traumatic memory episode remaining isolated and unassociated would thus not be indexed, reduced, and processed. The linking of the traumatic event would need to be carried out during wakefulness, for example by using imagery rehearsal therapy (Harb et al. 2012), so as potentially to modify both elaborative encoding and indexing. In the context of PTSD, Roisin $(2003 ; 2010)$ considers the exhortation of the desire to live as an essential action in the psychological growth of individuals having suffered a traumatic experience. When this desire resurfaces, post-traumatic symptoms disappear. Roisin therefore seems to link desire to elaborative encoding during wakefulness. Through its emotional salience, desire, like the dream, would contribute to emotional regulation.

Llewellyn's article suggests that we all have supermemorizer talents in our sleep, but not necessarily when awake. Explicit memorization that is largely superior to normal can be accompanied by emotional or social difficulties, like Asperger's syndrome of the autism spectrum (as in Daniel Tammet (Baron-Cohen et al. 2007)), or like savant syndrome (as in Kim Peek (Treffert \& Christensen 2005)). Moreover, memorizers and individuals with Asperger's syndrome would not think of these AAOM rules (Tammet 2007) because they do not explicitly use them. Rather, the rules would function autonomously during wakefulness, as they do during sleep in the general population. Perhaps forgetting most of our dreams would serve to preserve our mental resources for surrounding stimuli during wakefulness, so as to not be distracted by our inner world. Therefore, could forgetting our dreams be necessary to our cognitive and emotional balance? If the AAOM enables the regulation of emotions through catharsis or indexing, should it remain predominantly nonconscious so as not to hinder social relations?

In addition to remaining at a level of implicit memorization, the use of the AAOM during REM sleep should increase with the quantity of information that confronts the individual. We could imagine that children highly exposed to new information would require increased processing in order to strengthen anchoring and increase their ability to remember. As we know that REM sleep is more predominant in infants (Marcus et al. 2008), we could propose a developmental perspective on the necessity of assimilating a varying quantity of episodes (episodic memory) to retrieve. Thus, in addition to forgetting, the increase of anchoring in infants could be necessary to their cognitive and emotional development, particularly in the context of social relationships. 